\title{
Transkulturelle Psychotherapie
}

\section{Neue Perspektiven in der klinischen Anwendung}

\author{
Enver Cesko
}

Psychotherapie-Wissenschaft 8 (2) 51-56 2018

www.psychotherapie-wissenschaft.info

CC BY-NC-ND

https://doi.org/10.30820/8243.09

\begin{abstract}
Zusammenfassung: Dieser Artikel zeigt die Funktionsweise der Positiven und Transkulturellen Psychotherapie in verschiedenen transkulturellen Umgebungen auf. In diesem Artikel werden die psychodynamischen, humanistischen und integrativen Herangehensweisen in Situationen vorgestellt, in denen transkulturelle Fragen im Mittelpunkt stehen. Der Artikel legt dar, wie ein Psychotherapeut, obwohl er tief in der orientalischen, osmanischen Kultur verwurzelt ist, das Wissen und die Erfahrung, die aus dieser orientalischen Umgebung stammen, mit den wissenschaftlichen Fortschritten des Okzidents verbinden kann, um diese Probleme zu lösen. Arbeiten aus diesen beiden kulturellen Traditionen werden zitiert und veranschaulicht.
\end{abstract}

Schlüsselworte: Transkulturelle Psychotherapie, Positive Psychotherapie, psychodynamisch, humanistisch, integrativ, Globalisierung, Radikalisierung, Balance, Kultur, Werte

\section{Was genau ist transkulturelle Psychotherapie?}

Ich möchte mit einer Geschichte beginnen, die oft in der psychodynamischen und humanistischen Psychotherapie verwendet wird. Die Geschichte stammt aus dem Buch Der Kaufmann und der Papagei: Orientalische Geschichten in der Positiven Psychotherapie [Oriental Stories as a tools in Psychotherapy, The Merchant and the Parrot] (Peseschkian, 1986).

\section{Der Kaufmann und der Papagei}

Ein orientalischer Kaufmann hatte einen Papagei. Eines Tages kippte der Vogel eine Flasche Öl um. Der Händler wurde wütend und schlug den Papagei mit einem Stock auf den Hinterkopf. Nach diesem Vorfall konnte der Papagei, der zuvor grosse Intelligenz gezeigt hatte, nicht mehr sprechen. Es verlor Federn von seinem Schädel und wurde bald kahl auf dem Kopf. Eines Tages, als er auf der Kommode im Geschäft seines Herrn sass, betrat ein kahlköpfiger Kunde den Laden. Als er diesen Kunden sah, wurde der Papagei sehr aufgeregt. Er schlug mit den Flügeln, sprang hin und her, krächzte und begann dann zur Überraschung aller Anwesenden zu sprechen. «Hast du auch eine Flasche Öl umgeworfen und einen Schlag auf den Kopf bekommen, weil du auch keine Haare hast?» (Der persische Dichter Rumi, erzählt von Peseschkian, 1986, Epigraph S. v.).

In den letzten Jahrzehnten wurden verschiedene Begriffe mit ähnlicher Bedeutung wie «transkulturell» verwendet, zum Beispiel kulturell, kulturübergreifend, multikulturell, interkulturell, ethnisch, transkulturelle Gruppen und so weiter. In einigen Kontexten werden die Funktion, die Gruppendynamik und sogar die Bedeutung der diskutier- ten Interaktion durch die Sichtweise des jeweils präsentierenden Autoren und seiner Auswahl solcher Begriffe beeinflusst. In der Tat basieren diese verschiedenen Begriffe auf verschiedenen Bedeutungen, die es zu erkennen gilt, damit die von den KlientInnen und TherapeutInnen vorgebrachten Konzepte nicht herabgemindert werden.

Transkulturelle Psychotherapie bemüht sich in der Regel darum, Brücken zwischen den aus östlichen und westlichen Kulturen stammenden Werten und Einstellungen zu schlagen, auf die sich die Psychotherapie bezieht, unabhängig von der verwendeten Methode. Grund dafür ist, dass diese vertrauten Werte, Normen oder Symbole verfügbares Material sind, das verwendet werden kann, um die therapeutische Beziehung zwischen dem Patienten oder Klienten und dem Therapeuten aufzubauen. Wir akzeptieren diese Werte so, wie sie sind, um die ganzheitliche Sichtweise der humanistischen und psychodynamischen Psychotherapie zu vertreten.

Die psychologischen und psychotherapeutischen Methoden, die im späten 19. Jahrhundert in Europa und Amerika entstanden sind, haben ihre Wurzeln in der Renaissance. Sie haben zu sozialen und kulturellen Entwicklungen mit Betonung auf Prinzipien wie Individualismus, Demokratie, Radikalismus und Globalisierung geführt. Der Mensch arbeitet sich an Ideen ab, die sich auf das Selbst beziehen, wie zum Beispiel Selbstidentifikation und Selbstverwirklichung, Kernbegriffe in Psychologie und Psychotherapie.

Begriffe wie psychische Gesundheit, psychiatrische Störungen, emotionale Destabilisierungen, Stress, Trauma und andere psychische Erkrankungen übernehmen in einer therapeutischen Beziehung, in der KlientIn und TherapeutIn unterschiedlichen Ethnien, Kulturen, Rassen, Geschlechter, sozioökonomische wirtschaftliche Schichten und unterschiedliche Bildungshintergründe angehören, 
wichtige Merkmale konzeptioneller Relativität. Diese Konzepte, die sich aufgrund der verschiedenen Religionen, Rassen, Farben und Kulturen ergeben, bringen multikulturelle und transkulturelle Ansätze in die Psychotherapie. Basierend auf den Theorien der Psychoanalyse, der Erkenntnis- und Verhaltenstheorie und den Einflüssen von Ericksons psychosozialer Theorie und adlerianischer Sozialanalytik wurde in den 1970er Jahren die multikulturelle und transkulturelle Psychotherapie entwickelt (Lee \& Davis, 2000; Ramirez, 1999).

Ärzte der traditionellen mexikanischen Medizin führten eine qualitative Feldstudie durch, um die Wirksamkeit ethnotherapeutischer Praktiken von «curanderos» (Heilern) bei der Behandlung von Geisteskrankheiten zu bestimmen. Die Studie zeigte, dass die Behandlungspraktiken der mexikanischen HeilerInnen (curanderos) unter Einbeziehung von Spiritualität, veränderten Bewusstseinszuständen und die Bifokalität ritueller Interventionen signifikante Remissionen einiger psychiatrischer Störungen zeigten, einschliesslich Panikattacken, Abhängigkeitssyndrom und Schizophrenie (Zacharias, 2006).

Aus religiöser Perspektive werden psychische Probleme als spirituelle Probleme und deren Beseitigung als spirituelle Medizin angesehen. Die spirituelle Medizin kann jedoch nicht vollständig von der physikalischen Medizin (al-tibb al-jismani) getrennt werden, da der Mensch sowohl aus einer Seele als auch dem Körper besteht (Nurdeen \& Mansor, 2006).

Ethnotherapeutische Aspekte, die bei der Behandlung von psychischen Gesundheitsproblemen gelten, können mit Konzepten gleichgesetzt werden, die in der positiven Psychotherapie als interkulturelle Psychotherapie bekannt sind.

«Das Ziel einer solchen Therapie darf nicht sein, kulturelle, gruppenbezogene, familiäre und persönliche Unterschiede auszublenden oder auf sie zu verzichten. Es scheint vielmehr wichtig zu sein, sowohl individuelle Eigenschaften als auch kulturelle Einzigartigkeit zu verwirklichen, wenn andererseits Möglichkeiten zum Umgang mit auftretenden Konflikten vorhanden sind» (Peseschkian, 1980, S. 39).

Gemäss dem Psychologie-Wörterbuch umfasst transkulturelle Psychotherapie:

«Arten der psychodynamischen Psychotherapie, die kulturelles Bewusstsein und Sensibilität betonen, einschliesslich sozial definierter Ideale von Gefühlen, Psychodynamik und Handlungen. Innerhalb der psychiatrischen Gemeinschaft wird diese Terminologie in gewissem Sinne etwas häufiger als die multisoziale Beratung in der klinischen Psychologie verwendet» (Nugent, 2009, S. 425).

Ihr zugrundeliegendes Konzept einer zusammengehörigen Menschheit bietet eine ganzheitliche Akzeptanz und ein besseres Sinnverständnis für die verschiedenen
Werte, Einstellungen, Verhaltensweisen, Gedanken und Konzepte.

Viele psychodynamische und humanistische Psychotherapieansätze funktionieren in unterschiedlichen kulturellen Umgebungen und versuchen, ihre inhärenten Werte, Normen, Verhaltensweisen, Regeln und den Behandlungsprozess zu akzeptieren und zu respektieren. Der Behandlungsprozess basiert auf der Beziehungsform zwischen Klient und Therapeut, die innerhalb des Rahmens dieser kulturellen Parameter entsteht. Auf diese Weise sind wir Psychotherapeuten in der Lage, aufgrund unserer unterschiedlichen kulturellen Herkunft unsere eigenen beruflichen Erfahrungen, Fähigkeiten und Fähigkeiten zu nutzen, um Klienten mit unterschiedlichen kulturellen Hintergründen zu erreichen und eine wirksame Behandlung zu erzielen. Ich möchte verdeutlichen, dass der Schwerpunkt des Behandlungsprozesses nicht Krankheit oder Leiden ist, sondern die Person als Ganzes und ihr einzigartiges Potenzial. Wenn TherapeutIn den PatientenIn in erster Linie als Menschen mit einem Glaubenssystem, einer Lebensphilosophie sowie vielen Fähigkeiten betrachtet, die in der gegenwärtigen Umgebung und im dynamischen Prozess seiner Beziehung zur Gesellschaft genutzt werden können, dann geht der Dialog über kulturelle Unterschiede - auch solche zwischen KlientIn und TherapeutIn - hinaus und sie werden den Erfolg der Behandlung nicht behindern.

\section{Vier Dimensionen der transkulturellen Psychotherapie}

Heute leben wir in Umgebungen, die sehr unberechenbar und belastend sind und nicht genügend Sicherheit bieten. Einige von uns empfinden dies als interessante Zeit, eine Zeit, in der sich Wissenschaft, Technologie und Gesellschaft ständig mit extremen materialistischen Bestrebungen weiter entwickeln. Ein grosser Prozentsatz der Menschen versteht sich als Gläubige, die Spiritualität als wichtigen Aspekt ihres Lebensstils einsetzen. Es gibt oft signifikante Unterschiede in den Ansichten, Konzepten, Gewohnheiten und Verhaltensweisen zwischen KlientInnen und TherapeutInnen bezüglich ihrer Ansätze, mit Problemen, Schwierigkeiten, Störungen und Krankheiten umzugehen. Normalerweise berücksichtigt eine Seite den kulturellen Hintergrund der anderen Seite nicht genügend.

Es ist nur allzu wahr, dass eine kompetente Behandlung auf einem korrekten Verständnis des kulturellen Hintergrunds der PatientInnen basieren muss - es ist unmöglich, PatientInnen richtig zu verstehen, ohne ihre kulturellen Normen zu berücksichtigen: religiöse Überzeugungen und Glaubenssätze, Konzepte von Erkrankung, Methoden des emotionalen Ausdrucks, Sprache, Einstellungen und soziale Normen (Abu Baker \& Dwairy, 2003).

In meiner psychotherapeutischen Praxis verwende ich das Modell der positiven Psychotherapie und den Ansatz der Körperpsychotherapie. Ich versuche, meine KlientInnen nicht als Krankheitsfälle zu betrachten, sondern als ganzheitliche Menschen, die wirkliche Fähigkeiten haben 
(Peseschkian, 1980). Wenn sie sich «krank» fühlen, sind sie möglicherweise nicht in der Lage, diese Fähigkeiten in dem Ausmass zu entfalten, das sie sich wünschen, aber während des psychotherapeutischen Prozesses werden sie daran arbeiten, sich ihrer eigenen Fähigkeiten bewusst zu werden und so die «Krankheit» aufzulösen.

Daher möchte ich einige grundlegende Informationen über mein Modell mitteilen, indem ich die transkulturelle Psychotherapie erläutere.

Die Positive Psychotherapie basiert auf einem psychodynamischen Konzept mit einer humanistischen Konzeption des Menschen und einem transkulturellen Ansatz. Es handelt sich um eine ressourcenorientierte und konfliktzentrierte Methode, bei der Symptome als Zeichen ungelöster Konflikte angesehen werden, die aus der Vergangenheit, meist aus der Kindheit, stammen. Das Wort "positiv» im Zusammenhang mit der Positiven Psychotherapie leitet sich vom lateinischen Wort «Positum»ab, was sachlich gegebene Fakten bedeutet, und erlaubt TherapeutInnen, KlientInnen als eine jeweils ganze Persönlichkeit zu sehen, die Probleme und Herausforderungen, Störungen und Krankheiten bewältigen können (Peseschkian, 1983). Positive Psychotherapie befolgt eine ursprüngliche Verfahrensweise; sie verwendet eine Reihe von Grundbegriffen, die in der Alltagssprache formuliert sind, so dass sie von verschiedenen Kulturen, ethnischen Gruppen und Gemeinschaften während des therapeutischen Prozesses in der Beziehung zwischen TherapeutInnen und PatientInnen leicht verstanden werden können. Im transkulturellen Psychotherapieansatz bilden die Grundbegriffe einen Rahmen für den psychotherapeutischen Prozess und können dazu genutzt werden, die Kommunikation zwischen verschiedenen psychotherapeutischen Modellen mit unterschiedlichen kulturellen Einheiten zu fördern.

$\mathrm{Da}$ ich aus einem östlichen, traditionellen und religiösen Hintergrund komme, Kontakt mit Studenten habe, Vorträge halte und die grundlegenden Konzepte den Studenten oder Klienten vorstelle, muss ich alle bestehenden kulturellen Normen der westlichen Kultur respektieren und akzeptieren.

Wenn ich einen Freund aus einem östlichen Kulturkreis treffe, und er fragt: «Wie geht es dir?», bin ich enttäuscht, weil er nicht auch nach meiner Familie fragt. Denn für mich ist die Familie wichtiger als ich selbst. Wenn ich andererseits einen Freund aus einem westlichen Kulturkreis treffe und der Freund fragt: "Wie geht es dir?», gebe ich automatisch die Antwort: «Danke, gut. Und dir?»

Dies zeigt, wie durch unterschiedliche kulturelle Werte und Normen die Reaktionen und Verhaltensweisen von Menschen unterschiedlich eingeschätzt und bewertet werden. Es verdeutlicht, wie wichtig die unterschiedlichen Konzepte und Normen sind. Für den einen Menschen ist es wichtig, wie er oder sie sich in Bezug auf Karriere und Körper entwickelt (normalerweise mit westlichem kulturellen Hintergrund), für einen anderen Menschen (normalerweise mit östlichem kulturellen Hintergrund) werden Beziehungen, Kontakte und spirituelle Werte wahrscheinlich am wichtigsten sein. Die gesellschaftli- chen Veränderungen passieren sehr rasch, wenn jemand von einem extremen Standpunkt zu einer anderen extremen Einstellung wechselt. Aus diesem Grund nimmt das «menschliche Bewusstsein diese Veränderungen nur in begrenztem Masse wahr» (Peseschkian, 1980, S. 25), weshalb sich die Menschen in der Gesellschaft immer im Übergang von alt nach neu, von Ost nach West, von links nach rechts befinden, von unten nach oben, aber leider nie das Ziel erreichen. Das Gleichgewicht zu halten war in den letzten zwanzig Jahren die anstrengendste Aufgabe.

«Mit Zunahme der Weltbevölkerung entstehen andere Probleme: Lebensmittelverteilung, sozioökonomische Kräfte, Probleme mit MigrantInnen und Geflüchteten, Terrorismus, Atommächte usw. Diese Probleme des modernen städtischen Lebens werden besonders durch die nie dagewesene Zahl von Geflüchteten und MigrantInnen auf dem Weg zu besseren Lebensumständen in für sie fremden Kulturen verschärft. All dies führt zu neuen Einflüssen auf die Arbeitsteilung, in der sich die Menschheit von der Differenzierung zur Spezialisierung bewegt und in der sich die Rollen ständig ändern. Dies kann am besten in Kulturen beobachtet werden, die sich noch in politischen und sozioökonomischen Übergängen auf dem Weg zu Ihrer wahren Identität befinden. Darüber hinaus erleben viele Familien globale Veränderungen, wenn sie versuchen, neue Identitäten zu finden, da sie ihre ursprünglichen Familienstrukturen lockern. Schliesslich: «Als Folge dieser Veränderungen öffnen sich nationale, ethnische und kulturelle Gruppen gegenüber der Aussenwelt, d.h. gegenüber aussenstehenden Gruppierungen. Ein solcher Trend geht mit neuen Möglichkeiten, neuen transkulturellen Problemen einher»» (Peseschkian, 1980, S. 36f.).

Im Allgemeinen muss die transkulturelle Psychotherapie zwei Fragen behandeln, die für die Aufrechterhaltung der harmonischen Verbindung zwischen verschiedenen Kulturen wichtig sind. Die eine lautet, was haben alle Menschen gemeinsam, und die zweite ist, worin unterscheiden sie sich? Das kann man auch so verstehen, dass Menschen die ihnen gemeinsamen Ähnlichkeiten kennen und akzeptieren können, nicht aber die Unterschiede, bei denen ihre Standpunkte weit auseinander liegen. Ihre traditionellen Gedankenwelten stossen wegen ihrer unterschiedlichen Inhalte und Zielprojektionen gegeneinander und es stellt sich immer die Frage, wie sich diese traditionellen Welten mit den neuen Bedingungen, in denen sich die Menschen befinden, in Einklang bringen lassen (Peseschkian, 2005).

Eines der Instrumente der Positiven Psychotherapie sind orientalische Erzählungen. Sie bieten transkulturelle Perspektiven in Form von Sprichwörtern, Mythen und Fabeln, in denen sich der Patient allegorisch wiedererkennt und so eine neue Form von Selbstvertrauen und Sicherheit aufbauen kann. Geschichten, Weisheiten und transkulturelle Beispiele aus anderen Kulturen werden als respektierte und wertschätzende MediatorInnen (Vermittler) 
zwischen TherapeutInnen und PatientInnen eingesetzt. Sie ermutigen zur Konfliktlösung mithilfe von Fantasie und bieten mnemonische Hilfe oder Eselsbrücken für zukünftige Situationen (Peseschkian, 1988). Beispielsweise: Ein Orientale, der nach Hause kommt, entspannt sich mitten im Lärm und Geschwätz all seiner Familienmitglieder und Nachbarn; wohingegen ein Westler lieber allein und in einer rubigen Umgebung entspannen möchte.

Die psychotherapeutische Behandlung muss aufgrund des kulturellen Hintergrunds der KlientInnen gewählt werden, um den PatientInnen bei kulturellen Anpassungen helfen zu können. Für eine kulturell kompetente Psychotherapie werden moderne PsychotherapeutInnen gebraucht, die in der Arbeit mit gemischter ethnischer Herkunft und unterschiedlichen kulturellen Hintergründen erfahren sind. Moderne PsychotherapeutInnen müssen besondere Qualitäten wie kulturelle Sensibilität, kulturelles Wissen, kulturelle Empathie und kulturelle Einsicht entwickeln (Tseng \& Streltzer, 2001).

\section{Vom Radikalismus zum Globalismus in der Psychotherapie}

Individuelle Lebensstile und Handlungsmuster im täglichen Leben mit ihren zusammengefügten Konzepten, Gewohnheiten, Verhaltensweisen und Errungenschaften führen zu vielen Konflikten, oft aufgrund extremer Orientierungen, die von individuellen Fähigkeiten und Möglichkeiten herrühren. Wenn die primären und sekundären Fähigkeiten nicht in Harmonie sind, widmet sich der Mensch nur einer dieser Möglichkeiten (Arbeit, Religion, familiäre Bindung usw.) und ignoriert andere, was zu Missverständnissen, ideologischen Konflikten und radikalen Entscheidungen führt, wie erzwungene Spannungen, Konflikte, Gewalt, Terrorismus und Krieg. Der Frieden in der Welt ist erschüttert und die Menschheit ist aus dem eigenen Gleichgewicht geraten, am Arbeitsplatz, in der Beziehung zu Gesellschaft, Gemeinschaft und Familie, und wir sorgen uns wegen der unterschiedlichen Lebensphilosophien um die Zukunft.

\footnotetext{
«Die Fähigkeit, Ziele zu verwirklichen (Fähigkeit zu erkennen; sekundäre Fähigkeiten) und die Fähigkeit, emotional zu sein (Fähigkeit zu lieben; primäre Fähigkeiten) schliessen einander nicht aus, sondern ergänzen einander. Wir können uns also eine Utopie mit sozialen Bedingungen vorstellen, in der ein Mensch alle seine Fähigkeiten zu einer harmonischen Beziehung entwickeln kann» (Peseschkian, 1980, S. 109).
}

Deshalb ist der transkulturelle Ansatz wie ein roter «Faden» im psychotherapeutischen Prozess, «denn der transkulturelle Aspekt bietet auch Material, um die individuellen Konflikte zu verstehen» (Peseschkian, 2005, S. 102), zwischen KlientInnen und Gesellschaft und in allen anderen wichtigen Beziehungen im Leben. Dieser Aspekt besitzt vor allem eine ausserordentliche soziale Bedeutung, die wir in einer Vielzahl von Problemen widergespiegelt sehen: von Einwanderern, Entwicklungshilfen, Schwierigkeiten, die sich in der Umwelt mit Angehörigen anderer kultureller Systeme ergeben, Problemen transkultureller Partnerschaften, Vorurteilen und deren Überwindung sowie mit alternativen Modellen, die aus anderen Kulturen stammen. In diesem Zusammenhang können auch politische Themen genannt werden, die sich aus transkulturellen Situationen ergeben (Peseschkian, 2005).

\section{Nicht für das Ende - sondern für einen Anfang}

Das Konzept der transkulturellen Psychotherapie ist schwierig und wegen der Komplexität des Verständnisses von Wachstum und Entwicklung sowohl der KlientInnen als auch der TherapeutInnen in therapeutischen Situationen nicht einfach anzuwenden. Aus diesem Grund sind sich viele PsychotherapeutInnen der Fehler und Probleme bewusst, die in der transkulturellen Psychotherapie auftreten können. Broude Leonid (2011) hat in seinem Artikel «Psychotherapeutisches Setting in nichtwestlicher (arabischer) Kultur» sehr wichtige Forschungsarbeiten von verschiedenen Autoren gesammelt, die typische Fehler und Schwierigkeiten in der transkulturellen Psychotherapie erklären:

1. Die Fehler der PsychotherapeutInnen beim Verstehen des Verhaltens von PatientInnen in Situationen, in denen TherapeutInnen nicht mit den besprochenen kulturellen Merkmalen vertraut sind. TherapeutInnen missverstehen kulturelle Verhaltenskodizes und werten sie so als persönliche Eigenschaften des Individuums oder des Widerstands ( «Pathologisierung» kultureller Merkmale) oder - umgekehrt - kulturelle Überfrachtung ( «Über-Kulturalisierung») wirklicher psychischer Probleme.

2. Fehler der PatientInnen beim Verstehen des Verhaltens der PsychotherapeutInnen. Emotionale Neutralität, Vermeidung direkter Einmischung in das Leben der PatientInnen und Zurückhaltung gegenüber den PatientInnen werden als Gleichgültigkeit, Kälte und mangelnde Hilfsbereitschaft verstanden. Die Einhaltung von Zeitvorgaben und pünktliche Bezahlung wird als Mangel an Interesse, Gier und - wieder - mangelnde Hilfsbereitschaft wahrgenommen.

3. Schwierigkeiten in Bezug auf Zweck und Ziel der Behandlung: Das Hauptinstrument (und das Zwischenziel) der dynamischen Psychotherapie ist das Verstehen der unbewussten Impulse sowie Korrekturen von Verhaltensweisen. Die östliche Gesellschaft bestraft den Ausdruck sexueller Wünsche (das heisst, das Bewusstwerden einer verheirateten Frau für ihre Zuneigung zu einem anderen Mann) oder die Wut auf die Eltern - ein Bewusstwerden dieser unbewussten Wünsche wird PatientInnen wahrscheinlich nicht glücklich machen. Darüber hinaus löst das Bewusstsein für interne Konflikte und deren Umsetzung in der Regel den Konflikt nicht und könnte ihn noch komplizierter machen (Broude, 2011). 
Was kann man also tun, um typische Fehler und Probleme zu vermeiden? Aus meiner persönlichen Erfahrung in der Arbeit in verschiedenen Ländern mit KlientInnen aus verschiedenen Kulturen habe ich gelernt, vertrauensvolle Beziehungen zwischen KlientInnen und TherapeutInnen aufzubauen. Jeder Psychotherapeut und jede Psychotherapeutin müssen vier Aspekte dieser Beziehung berücksichtigen, um Fehler zu vermeiden.

Der erste Aspekt ist die Akzeptanz der gesamten Persönlichkeit des Klienten bzw. der Klientin, unabhängig von Geschlecht, Rasse, Nationalität, Geschlecht, Glauben und traditionellen Werten. Dieser Aspekt umfasst, wie die Persönlichkeitsstruktur von der frühen Kindheit bis zum Erwachsenenalter gebildet wurde und sich entwickelte.

Der zweite Aspekt ist die Akzeptanz der Inhalte, die der KlientInnen oder PatientInnen in die Sitzung einbringen. Das Besprechen sensibler Themen während der Sitzung desorientiert oder stört oft den Behandlungsprozess durch ihren starken Einfluss auf TherapeutInnen oder KlientInnen. Die Reife und berufliche Weiterentwicklung der TherapeutInnen ist sehr wichtig, um eine Ablenkung des Behandlungsprozesses zu verhindern, so dass er nicht feststeckt oder die psychotherapeutische Verbindung nicht beschädigt wird. Mit dieser Art von Inhalten treten sehr oft Übertragung und Gegenübertragung auf und können bewusst oder unbewusst zu einem der Probleme werden, die die therapeutische Allianz stören.

Der dritte Aspekt ist die Perspektive der KlientInnen und die Interpretation der Sozial-und Familienstruktur durch die TherapeutInnen. Der Familienhintergrund, der sich aus verschiedenen ideologischen Glaubenskonzepten (Traditionalismus, Patriarchat, zeitgenössisch, ländlich oder städtisch, neuer Lebensstil usw.) entwickelt hat, kann die bestehenden und bereits starren Konzepte des Therapeuten beeinflussen und dies könnte ein erfolgreiches Ergebnis des Behandlungsprozesses in Zweifel ziehen. Manchmal sind sich TherapeutInnen nicht bewusst, dass sie in ihren tatsächlichen starren Konzepten als Folge von fest verwurzelten Einflüssen des sozialen und familiären Umfelds «blind» sind.

Der letzte und vierte Aspekt ist das bumanistische Konzept der Menschheit. Um die Glaubenssysteme, Lebensphilosophien sowie unterschiedliche Konzepte in Gesellschaft und Familiensystem zu verstehen und nachzuvollziehen, müssen TherapeutInnen die Sicht der PatientInnen auf die Welt und seinen Platz im kulturellen Kontinuum verstehen. Dies bedeutet, dass TherapeutInnen die eigene Qualität seiner psychologischen Betreuung entwickeln, korrigieren und verbessern muss, um unbewusste Vorurteile angemessen einzuschätzen, indem er ständig mögliche Fehler hinterfragt.

\section{Schlussfolgerungen}

Der Einfluss der Kultur auf die Psychotherapie wird vor allem aus praktischen und theoretischen Perspektiven verstanden, die durch die unterschiedlichen kulturellen Hintergründe bestimmt sind, die zwischen KlientInnen und TherapeutInnen bestehen können. Im psychotherapeutischen Prozess besteht die Rolle der PsychotherapeutInnen darin, sich der bestehenden Unterschiede zwischen KlientIn und TherapeutIn bewusst zu sein: «Wenn es nötig ist, PatientInnen zu helfen, den Sinn des Lebens, die Implikationen der Lösung von Problemen im Leben und die Richtung, in der sich PatientInnen im Leben bewegen, zu bewerten» (Tseng \& Streltzer, 2001, S. 34).

Heute betrachten sich immer mehr PsychotherapeutInnen als "aufgeschlossen» und wenden eklektische Therapieansätze an, die am besten zu den kulturellen Hintergründen, Werten, Konzepten und der Persönlichkeit der PatientInnen passen. Die sprachlichen Unterschiede stellen oft Hindernisse im therapeutischen Prozess dar. Darüber hinaus können religiöse Überzeugungen ein Schlüssel für Störungen und Scheitern der Kommunikation in der ethnopsychotherapeutischen Beziehung sein. In dieser sind psychologische Interventionen und erforderliches kulturelles Wissen und kultureller Dialog für die erfolgreiche Behandlung überaus wichtig (White, 1999). Einige TherapeutInnen, die in einer grösseren Gesellschaft arbeiten, wenden bei ihren KlientInnen einen eklektischen Ansatz an und berücksichtigen dabei, dass jeder Klient und jede Klientin seine bzw. ihre eigene Kultur haben. Einige TherapeutInnen wenden bestimmte Methoden und Prinzipien innerhalb verschiedener Hintergründe in Bezug auf Minderheiten (Aponte et al., 1995), Gesellschaftsschichten (Foster et al., 1996), sexuelle Orientierungen (Greene, 1997) oder körperliche Behinderungen an. Solche Patienten können sich in ihren Lebenserfahrungen und Sichtweisen von der Welt so sehr von den Therapeuten unterscheiden, dass Empathie über diese Barriere der «Unterschiede» hinweg erforderlich ist (Tseng \& Streltzer, 2001).

Die Transkulturelle Psychotherapie wird auch zu einem integrativen und ganzheitlichen Ansatz, wenn Krisen, Probleme und Schwierigkeiten nicht nur für Psychotherapeuten, sondern auch für Einzelpersonen in der Gesellschaft, Politiker in der Regierung und religiöse Führer zu Themen von Interesse werden. Sie müssen sich dann zusammentun, um gemeinsame Konzepte für den Umgang mit diesen Themen zu finden und die Probleme zu lösen. Um ein weises Sprichwort zu zitieren: «Kleine Handlungen verändern die Welt; wenn du mit kleinen Dingen nicht geduldig bist, kannst du keine grossen Dinge tun» (Peseschkian, 2005, S. 18).

\section{Literatur}

Abu Baker, K. \& Dwairy, M. (2003). Cultural Norms Versus State Law in Treating Incest: A Suggested Model for Arab Families. Child Abuse and Neglect, 27, 109-123. In Broude, L. (2011), Psychotherapeutic Setting in Non-Western (Arabic) Culture. World Journal Psychotherapy, 1(4), pp. 33 (Development Psychotherapy). Aponte, J.F., Rivers, R.Y. \& Wohl, J. (Eds.). (1995). Psychological Interventions and Cultural Diversity. Boston: Allyn \& Bacon. In Tseng, W.-S.H. \& Streltzer, J. (Eds.). (2001), Culture and Psychotherapy. A Guide to Clinical Practice (pp. 266). Washington D. C., London: American Psychiatric Press. 
Broude, L. (2011). Psychotherapeutic Setting in Non-Western (Arabic) Culture. World Journal Psychotherapy, 1(4), 33 (Development Psychotherapy).

Foster, R.M.P., Moskowitz, M. \& Javier, R.A. (Eds.). (1996). Reaching Across Boundaries of Culture and Class: Widening the Scope of Psychotherapy. Northvale/NJ: Jason Aronson. In Tseng, W.-S.H. \& Streltzer, J. (Eds.). (2001), Culture and Psychotherapy. A Guide to Clinical Practice (pp. 266). Washington D. C., London: American Psychiatric Press.

Greene, B. (Ed). (1997). Ethnic and Cultural Diversity Among Lesbians and Gay Men. Thousand Oaks, CA: Sage. In Tseng, W.-S.H. \& Streltzer, J. (Eds/Hrsg.). (2001), Culture and Psychotherapy. A Guide to Clinical Practice (pp. 266). Washington D.C., London: American Psychiatric Press.

Lee, R. M. \& Davis, C. III. (2000). Cultural orientation, past multicultural experience, and a sense of belonging on campus for Asian American college students. Journal of College Student Development, 41, 110-114.

Matsumuto, D. (Ed./Hrsg.). (2009). The Cambridge Dictionary of Psychology. Edinburgh, Cambridge/UK: Cambridge University Press.

Nugent, Pam M.S. (2013). Transcultural Psychotherapy. https:// psychologydictionary.org/transcultural-psychotherapy/ (29. April 2013).

Nurdeen, D. \& Mansor, A.T. (2005). Mental health in Islamic medical tradition. The International Medical Journal, 4(2), 76-79.

Peseschkian, N. (1980). Positive Family Psychotherapy, the Family as Therapist. Berlin: Springer.

Peseschkian, N. (1983). In Search of Meaning, A Psychotherapy of Small Steps. Berlin: Springer

Peseschkian, N. (1986). Oriental Stories as Tools in Psychotherapy. Berlin: Springer.

Peseschkian, N. (1988). Positive Psychotherapy, Theory and Practice of a New Method. Berlin: Springer.

Peseschkian, N. (2005). If You Want Something You Never Had, Then Do Something You Never Did. New Delhi: New Dawn Press Group.

Ramirez, M. (1999). The Multicultural Model of Psychotherapy and Counseling. Multicultural Psychotherapy. e-Book 2017 International Psychotherapy Institute.

Tseng, W.-S.H. \& Streltzer, J. (Eds.). (2001). Culture and Psychotherapy. A Guide to Clinical Practice. Washington D. C., London: American Psychiatric Press.

White, T. A. (1999). «Ethnopsychology: A Breakdown in Communication». Honors Theses. Paper 122. Southern Illinois University Carbondale OpenSIUC.

Zacharias, S. (2006). Mexican Curanderismo as Ethnopsychotherapy: A qualitative study on treatment practices, effectiveness, and mechanisms of change. International Journal of Disability, Development and Education, 53(4), 381-400. Issue 4: (Ausg. 4) Indigenous and Complementary and Alternative Healing Practice.

\section{Psychothérapie transculturelle. \\ De nouvelles perspectives dans l'application clinique}

Cet article démontrera la fonctionnalité de la Psychothérapie Positive et transculturelle dans différents environnements transculturels. Cet article présentera les approches psychodynamiques, humanistes et intégratives dans des situations dans lesquelles les questions transculturelles sont la question en débat. L'article montrera comment un psychothérapeute, bien que profondément ancré dans la culture orientale, ottomane, peut fusionner la connaissance et l'expérience recueillie dans cet environnement oriental avec les avancées scientifiques de l'occident pour résoudre ces problèmes. Des travaux de ces deux traditions culturelles seront cités et illustrés.

Mots-clés : psychothérapie transculturelle, psychothérapie positive, psychodynamique, humaniste, intégrative, globalisation, radicalisation, équilibre, culture, valeurs

\section{Psicoterapia transculturale. Nuove prospettive nell'applicazione clinica}

Il presente articolo dimostrerà la funzione della psicoterapia positiva e transculturale in diversi ambienti transculturali. L'articolo presenterà gli approcci psicodinamici, umanistici e integrativi a situazioni in cui le domande transculturali sono l'elemento in questione. L'articolo mostrerà come lo psicoterapeuta, sebbene profondamente radicato nella cultura orientale ottomana, può unire le conoscenze e le esperienze derivanti da questo ambiente con i progressi scientifici occidentali al fine risolvere tali problemi. Saranno citati e illustrati lavori di entrambe queste tradizioni culturali.

Parole chiave: psicoterapia transculturale, psicoterapia positiva, psicodinamica, umanistica, integrativa, globalizzazione, radicalizzazione, equilibrio, cultura, valori

\section{Der Autor}

Enver Cesko ist Psychologe und Psychotherapeut. Er ist Präsident und Gründer der Kosovo Association for Psychotherapy und Vorstandsmitglied der European Association for Psychotherapy (EAP), der World Association of Positive Psychotherapy (WAPP), des World Council of Psychotherapy (ECP) und der European Association for Body Psychotherapy (EABP). Nach dem Krieg im Kosovo wurde er in Washington D.C. in MindBody Medicine (Gedanken-Körper-Medizinkonzept) ausgebildet, um als Traumatherapeut mit Menschen zu arbeiten, die unter Kriegstraumata leiden. Nach seiner Dozententätigkeit an verschiedenen Universitäten arbeitet er aktuell in seiner eigenen Praxis in Pristina, Kosovo, und führt regelmässig internationale Fortbildungen (Trainings) in Positiver Psychotherapie in verschiedenen Ländern durch. Er präsentierte seine Workshops und Vorträge auf mehr als 20 Welt- und Europa-Kongressen für Psychotherapie und veröffentlichte mehr als 150 verschiedene Artikel in Fach- und Wochenzeitschriften.

\section{Kontakt}

envercesko@hotmail.com 